\title{
Evaluation of changes in the activity of proteolytic enzymes and their inhibitors in the processes that accompany the growth of gastric cancer
}

\author{
Wojciech Kielan ${ }^{1}$, Janusz Suzanowicz ${ }^{1}$, Maciej Siewiński², Yousif Saleh ${ }^{2}$, Anna Janocha ${ }^{3}$, Adam Skalski ${ }^{1}$, \\ and ROBERT TARNAWA ${ }^{1}$ \\ ${ }^{1}$ Second Department of General and Oncological Surgery, Wroclaw Medical University, Wroclaw, Poland \\ ${ }^{2}$ First Department of Gynaecology and Obstetrics, Wroclaw Medical University, ul. Chałubińskiego 3, 50-368 Wroclaw, Poland \\ ${ }^{3}$ Department of Physiology, Wroclaw Medical University, Wroclaw, Poland
}

\begin{abstract}
Background. Many investigators have observed a correlation between the aggressiveness of malignant tumor growth and the levels of cysteine peptidases and their autogenous inhibitors. Cathepsins $B$ and $L$ are activated by pepsin in an acidic pH. This fact encouraged us to measure the activity of these enzymes in tissue samples of gastric cancer.

Methods. We measured the activities of cathepsins $B$ and $L$, and their precursors and inhibitors, in homogenates of tissue samples obtained from operations for gastric cancer. We compared the results for the homogenates of tissues from three different sites: the tumor center, the zone of cancer invasion (border of the tumor), and healthy tissue.

Results. The highest activities of free cysteine peptidases and those in complexes with their inhibitors, as well as their precursors, were observed in the border of the tumor, while the activities in healthy tissue were significantly lower.

Conclusion. The local activities of cysteine peptidases and their inhibitors reflect the topographical differences between the center of the tumor, the zone of invasion, and healthy tissues in gastric cancer patients. In addition, the results for the pattern of changes in the activity of cysteine peptidases according to the degree of tissue infiltration were not dependent on the method of measurement (colorimetry vs spectrofluorometry).
\end{abstract}

Key words Cysteine peptidases - Cathepsin B - Cathepsin L . Cysteine peptidase inhibitors $\cdot$ Gastric cancer

\section{Introduction}

The enzymatic processes that accompany the growth of gastric cancer catalyze the destruction of normal tissues. It has been found that mostly specific proteolytic enzymes participate in such processes, produced either in the cells of the malignant tumor, or in normal cells

Offprint requests to: $\mathrm{M}$. Siewiński

Received: December 12, 2002 / Accepted: September 24, 2003 situated in the zone of tumor invasion [1,2]. The understanding of changes in the activities of these proteolytic enzymes that initiate carcinogenesis has led to the concept of a cascade of mutual activation of proteolytic enzymes, which determines the ongoing activation of enzymes that take part in the crucial processes of carcinogenesis [3]. These changes are initiated by the presence of active cathepsin $\mathrm{D}$ or pepsin, which in acidic $\mathrm{pH}$, activate precursors of cysteine endopeptidases. During malignant invasion, cathepsin B is initially produced in its precursor form, and the activity of this enzyme is stimulated by cathepsin D or pepsin in a low $\mathrm{pH}$. Both low $\mathrm{pH}$ and high pepsin activity are present in the stomach, thus providing optimal conditions for the activation of cysteine peptidase precursors. The precursor enzymes are produced within cancer cells and only after secretion to the surrounding tissues are they activated and catalyze tumor invasion $[4,5]$. Of all cysteine peptidases (EC 3.4.22) mainly cathepsins B and L are involved in the metabolism of malignant tumors; these subsequently catalyze the conversion of other peptidases into their active forms, which are responsible for the degradation of normal tissues, and also for bringing about tumor invasion and metastases [5,6]. It has been confirmed that active cathepsin B is associated with many key processes in tumor growth; this finding was achieved by measurements of its activity in tissues and serum of people hospitalized for gastric cancer $[7,8]$.

A positive correlation between tumor invasiveness, as well as its metastatic potential, and the secretion of cysteine peptidases (particularly cathepsins B and L) has been well documented in the literature on this subject $[3,9]$. We postulate that cysteine peptidase activity could be used as a marker of cancer aggressiveness in diagnostic procedures in oncology. The correlation between the progression of early gastric cancer and the activity of cathepsins $\mathrm{B}$ and $\mathrm{L}$ has been studied by Dohchin et al. [10]. Their results correlated well with immunohistology and it was confirmed that the level of 
these enzymes can be considered a marker of invasion and aggressiveness of gastric cancer.

The aim of our study was to analyze the activity of proteolytic enzymes (cysteine peptidases), and their precursors and autogenous inhibitors, in biopsies of the center of tumor, the zone of invasion, and healthy tissue of the stomach in patients with advanced diffuse type gastric cancer. Additionally, another aim was to compare the above results obtained by means of two different methods of measurement, i.e., colorimetry vs spectrofluorometry.

\section{Patients, materials, and methods}

\section{Patients and samples}

The study was carried out from January 1998 to March 2000 with the homogenates of samples of resected tissues taken from 29 patients operated on for advanced gastric cancer at the Second Department of General and Oncological Surgery, Wroclaw Medical University. The group included 21 men and 8 women, aged from 28 to 73 . In 20 patients, total gastric resection was performed, and subtotal resection was done in 9. All cases were advanced gastric carcinoma, diffuse Lauren type.

After the operation, three tissue samples, about $0.5 \mathrm{~g}$ each, were taken from the resected stomach of each patient: (a) from the center of the tumor, (b) from the border of the tumor (zone of invasion), and (c) from visually normal healthy tissues, distant from the tumor. The samples were initially washed and stored frozen in $1.0 \mathrm{ml}$ of normal saline at a temperature of $-20^{\circ} \mathrm{C}$ for about 1 month.

\section{Sample preparation}

The following measurements were made in homogenates of these tissue samples: active cysteine peptidases (CP), and their precursors (pCP) and inhibitors, the latter being measured as three forms: active inhibitors of cysteine peptidases $\left(\mathrm{ICP}^{37}\right)$, the total pool of inhibitors produced by the patient's tissues $\left(\mathrm{ICP}^{80}\right)$, and the inhibitors bound in enzyme-inhibitor complexes $(\triangle \mathrm{ICP})$.

For this purpose, thawed tissue samples were homogenized for $20 \mathrm{~min}$ in normal saline, in the proportion of $1.0 \mathrm{~g}$ tissue in $6.0 \mathrm{ml}$ saline, with a homogenizer MPW309 (Universal Laboratory Aid, Warsaw, Poland). The homogenates were centrifuged for $30 \mathrm{~min}$ at a temperature of $4^{\circ} \mathrm{C}$, at a speed of $10000 \mathrm{~g}$, using a Sorvall RC-5B centrifuge (Sorvall, Hamburg, Germany). The supernatant was separated, frozen at $-20^{\circ} \mathrm{C}$, and then stored at $20^{\circ} \mathrm{C}$ for not longer than 1 month. When thawed, the clear solutions served for biochemical examinations.

\section{Chemical reagents}

The following reagents were used in the study: N-benzoyl-DL-arginyl- $\beta$-naphthylamide (BANA), benzoyl-phenyl-arginyl-aminocoumarin (Z-Phe-ArgAMC), 7-amino-4-methylcoumarin (Mec), inhibitor E64 , and enzymes. Papain and pepsin were manufactured by Sigma (St. Louis, MO, USA); methylamine $40 \%$ solution and dimethyl sulfoxide (DMSO) were manufactured by Fluka BioChemika (Birchs, Switzerland).

\section{Measurement of enzyme activities}

In the recent literature on the subject the activities of cysteine peptidases have been measured by two equivalent methods: spectrofluorometry and colorimetry. Most of our measurements were made by the colorimetric method, but we also used spectrofluorometry to prove that the results were not dependent on the method used.

\section{Measurement of the activity of cysteine peptidases by spectrofluorometry}

The activity of cysteine peptidases was measured with a Perkin Elmer UV/VIS Spectrometer Lambda Bio 20 (Perkin Elmer, Wellesley, MA, USA). One sample contained $100 \mu$ of supernatant from the homogenized tissue, diluted with normal saline as needed according to the concentration of the enzyme; $700 \mu l 0.4 \mathrm{M}$ phosphate buffer at $\mathrm{pH} 6.0$, including $4 \mathrm{mM}$ ethylenediamine tetraacetic acid (EDTA), $2.5 \mathrm{mM}$ dithiothreitol (DTT), and $200 \mu \mathrm{l}$ diluted substrate concentrate Z-Phe-Arg-N$\mathrm{Mec}$, whose concentration was $40 \mu \mathrm{M}$.

The sample was incubated at $37^{\circ} \mathrm{C}$ for $60 \mathrm{~min}$. The reaction of hydrolysis was terminated by adding $2.0 \mathrm{ml}$ of $1.0 \mathrm{mM}$ iodoacetic acid to each sample. Fluorescence of the liberated 7-amino-4-methylcoumarin (Mec) was measured using the wavelength $\lambda=370 \mathrm{~nm}$ for excitation and $\lambda=440 \mathrm{~nm}$ for emission. The measurements were made in regard to baseline solution, which differed from the analyzed samples in that $2.0 \mathrm{ml}$ of iodoacetic acid was added before the substrate. The results are given in units of activity of cysteine endopeptidases, calculated for the amount of protein appearing in the sample. One unit of enzyme activity was defined as that amount of the enzyme that would liberate $1 \mathrm{nM}$ of Mec per $1 \mathrm{~min}$, calculated for $1 \mathrm{mg}$ of protein [11].

\section{Measurement of the activity of precursors of cysteine peptidases}

To the diluted supernatant samples of tissue homogenates $(50 \mu \mathrm{l}$ each), $150 \mu \mathrm{l}$ of pepsin, diluted to $0.7 \mathrm{mg} / \mathrm{ml}$ in $0.01 \mathrm{M}$ acetate buffer at $\mathrm{pH} 3.0$, was added. The samples were incubated at $37^{\circ} \mathrm{C}$ for $60 \mathrm{~min}$, then 
$0.4 \mathrm{M}$ phosphate buffer at $\mathrm{pH} 6.0$, containing $4 \mathrm{mM}$ EDTA and $200 \mu \mathrm{l}$ of diluted substrate concentrate ZPhe-Arg-N-Mec, whose concentration was $40 \mu \mathrm{M}$, were added to make a volume of $1.0 \mathrm{ml}$. The samples were then incubated at $37^{\circ} \mathrm{C}$ for $60 \mathrm{~min}$ and the active cysteine endopeptidases were measured as described above. The activity of the precursors was calculated as the difference between enzyme activities measured directly in the homogenate and those measured after preincubation with pepsin [4].

\section{Measurement of the activity of cysteine peptidases by colorimetry}

To the samples of homogenate ( $10 \mu \mathrm{l}$ to $100 \mu \mathrm{l}$ each) $50 \mu \mathrm{l}$ of $0.66 \mathrm{mM}$ BANA substrate was added, then $0.01 \mathrm{M}$ phosphate buffer at $\mathrm{pH} 6.0$, containing BANA, was added, and after $12 \mathrm{~h}$ of preincubation at $37^{\circ} \mathrm{C}$, free $\beta$-naphthylamine was measured by Barrett's method. One unit of enzyme activity of cysteine proteinases was defined as that amount of the enzyme that would liberate $1 \mathrm{nM}$ of $\beta$-naphthylamine per $1 \mathrm{~h}$, calculated for $1 \mathrm{mg}$ of protein [12].

\section{Measurement of the activity of cysteine peptidase inhibitors by colorimetry}

Before the measurement of the inhibitors of cysteine peptidases, $\alpha_{2}$-macroglobulin was inactivated [13]. To measure the active inhibitors of cysteine endopeptidases $\left(\mathrm{ICP}^{37}\right)$, the analyzed homogenate samples were incubated with papain solution, and after $30 \mathrm{~min}$, the amount of liberated $\beta$-naphthylamine was measured. The procedure was as follows:

1. Samples of homogenate $(50-\mu l)$ were incubated in $0.6 \mathrm{ml}$ of $0.02 \mathrm{M}$ phosphate buffer containing $2 \mathrm{mM}$ EDTA and $2 \mathrm{mM}$ L-cysteine at $\mathrm{pH}$ 6.0. Then $50 \mu \mathrm{l}$ of papain solution in the same buffer was added, the sample was incubated at $37^{\circ} \mathrm{C}$ for $10 \mathrm{~min}$, and liberated $\beta$-naphthylamine was calculated from the standard titration curve prepared earlier. One unit of inhibitor activity was defined as that amount of the inhibitor that would inhibit 1 unit of papain activity.

2. The total amount of inhibitors $\left(\mathrm{ICP}^{80}\right)$ was measured after preincubation of the samples of homogenate supernatant in glycine buffer at $\mathrm{pH} 2.0$, for $20 \mathrm{~min}$ at $80^{\circ} \mathrm{C}$. Then the activity of inhibitors of cysteine peptidases was measured in the presence of BANA substrate, as described above for the measurement of free inhibitors $\left(\mathrm{ICP}^{37}\right)$.

3. The activity of inhibitors bound in complexes was calculated as the difference between total and free inhibitors: $\triangle \mathrm{ICP}=\mathrm{ICP}^{80}-\mathrm{ICP}^{37}[14]$. The enzyme activities and the levels of inhibitors were calculated for $1 \mathrm{mg}$ of protein $[13,14]$.

The concentration of protein in the analyzed samples was measured by a method using Bradford's reagent, where pure bovine albumin was taken as the standard [15].

\section{Results}

The measurements of these enzymatic markers of tumor growth enabled us to compare the individual differences in their activities for each patient. We compared the same parameters between patients, as well as the differences between the sampling sites in each individual patient (Tables 1 and 2, Figs. 1-6). In 17 patients, the highest activities of cysteine peptidases (CP) and their precursors $(\mathrm{pCP})$ and inhibitors in complexes with enzymes $(\triangle \mathrm{ICP})$ were found in the homogenates of cancer tissue, whereas in the remaining 12 patients, these activities were highest in the homogenates of tissue from the zone of invasion. In the whole series, the lowest mean activities of cysteine peptidases and their precursors and enzyme-inhibitor complexes were found in the homogenates of healthy tissue (Table 1). For the spectrofluorometric measurements, it was noted that the highest activities of cysteine peptidases and their precursors were present in cancer tissue in 22 patients, while in 7 patients they were highest in the tissue from the border of malignant growth (Table 2).

Table 1. Activities of cysteine peptidases, and their precursors and inhibitors, as determined by colorimetry (Col) and spectrofluorometry $(\mathrm{F})$ in gastric cancer tissues

\begin{tabular}{|c|c|c|c|c|c|c|c|}
\hline Location of tissue sample & & $\mathrm{CP}(\mathrm{Col})$ & $\mathrm{ICP}^{37}$ & $\mathrm{ICP}^{80}$ & $\Delta \mathrm{ICP}$ & $\mathrm{CP}(\mathrm{F})$ & $\mathrm{pCP}(\mathrm{F})$ \\
\hline \multirow[t]{2}{*}{ (a) Center of tumor } & Mean & 0.447 & 1.118 & 1.611 & 0.493 & 10.170 & 5.700 \\
\hline & SD & 0.139 & 0.108 & 0.185 & 0.076 & 8.217 & 3.988 \\
\hline \multirow[t]{2}{*}{ (b) Zone of invasion } & Mean & 0.397 & 0.928 & 1.295 & 0.368 & 3.325 & 4.260 \\
\hline & SD & 0.127 & 0.078 & 0.039 & 0.117 & 2.157 & 3.507 \\
\hline \multirow[t]{2}{*}{ (c) Healthy tissue } & Mean & 0.305 & 0.752 & 0.861 & 0.110 & 1.480 & 0.825 \\
\hline & SD & 0.025 & 0.399 & 0.423 & 0.023 & 0.863 & 0.290 \\
\hline
\end{tabular}

$\mathrm{CP}$, cysteine peptidases; pCP, precursors; $\mathrm{ICP}^{37}$, active inhibitors; $\mathrm{ICP}^{80}$, total pool of inhibitors; $\triangle \mathrm{ICP}$, inhibitors bound in enzyme-inhibitor complexes 
Table 2. Individual activities of cysteine peptidases (CP), and their precursors (pCP) and inhibitors (ICP), as determined by colorimetry $(\mathrm{Col})$ and spectrofluorometry $(\mathrm{F})$ in gastric cancer tissues

\begin{tabular}{|c|c|c|c|c|c|c|c|}
\hline Patient no. & Tissue sample & $\mathrm{CP}(\mathrm{Col})$ & $\mathrm{ICP}^{37}$ & $\mathrm{ICP}^{80}$ & $\Delta \mathrm{ICP}$ & $\mathrm{CP}(\mathrm{F})$ & $\mathrm{pCP}(\mathrm{F})$ \\
\hline I & $\begin{array}{l}1 \mathrm{a} \\
2 \mathrm{~b} \\
3 \mathrm{c}\end{array}$ & $\begin{array}{l}0.545 \\
0.487 \\
0.322\end{array}$ & $\begin{array}{l}1.195 \\
0.873 \\
0.469\end{array}$ & $\begin{array}{l}1.742 \\
1.323 \\
0.562\end{array}$ & $\begin{array}{l}0.547 \\
0.450 \\
0.093\end{array}$ & $\begin{array}{l}4.36 \\
1.80 \\
0.87\end{array}$ & $\begin{array}{l}2.88 \\
1.78 \\
1.03\end{array}$ \\
\hline II & $\begin{array}{l}4 \mathrm{a} \\
5 \mathrm{~b} \\
6 \mathrm{c}\end{array}$ & $\begin{array}{l}0.687 \\
0.436 \\
0.430\end{array}$ & $\begin{array}{l}0.513 \\
1.697 \\
0.733\end{array}$ & $\begin{array}{l}1.272 \\
2.018 \\
0.847\end{array}$ & $\begin{array}{l}0.760 \\
0.321 \\
0.114\end{array}$ & $\begin{array}{r}21.91 \\
4.09 \\
0.73\end{array}$ & $\begin{array}{r}32.45 \\
5.12 \\
0.98\end{array}$ \\
\hline III & $\begin{array}{l}7 \mathrm{a} \\
8 \mathrm{~b} \\
9 \mathrm{c}\end{array}$ & $\begin{array}{l}0.765 \\
0.809 \\
0.521\end{array}$ & $\begin{array}{l}1.318 \\
1.863 \\
0.245\end{array}$ & $\begin{array}{l}2.294 \\
2.733 \\
0.585\end{array}$ & $\begin{array}{l}0.976 \\
0.870 \\
0.340\end{array}$ & $\begin{array}{l}8.07 \\
3.25 \\
1.15\end{array}$ & $\begin{array}{r}12.67 \\
9.65 \\
3.61\end{array}$ \\
\hline IV & $\begin{array}{l}10 \mathrm{a} \\
11 \mathrm{~b} \\
12 \mathrm{c}\end{array}$ & $\begin{array}{l}0.876 \\
0.395 \\
0.309\end{array}$ & $\begin{array}{l}1.861 \\
0.634 \\
0.110\end{array}$ & $\begin{array}{l}2.291 \\
1.045 \\
0.331\end{array}$ & $\begin{array}{l}0.430 \\
0.411 \\
0.221\end{array}$ & $\begin{array}{l}6.32 \\
5.69 \\
0.89\end{array}$ & $\begin{array}{l}2.11 \\
4.76 \\
0.76\end{array}$ \\
\hline $\mathrm{V}$ & $\begin{array}{l}13 \mathrm{a} \\
14 \mathrm{~b} \\
15 \mathrm{c}\end{array}$ & $\begin{array}{l}0.298 \\
0.365 \\
0.287\end{array}$ & $\begin{array}{l}0.064 \\
0.531 \\
0.321\end{array}$ & $\begin{array}{l}0.601 \\
1.101 \\
0.552\end{array}$ & $\begin{array}{l}0.537 \\
0.570 \\
0.231\end{array}$ & $\begin{array}{l}0.44 \\
4.89 \\
1.32\end{array}$ & $\begin{array}{l}0.69 \\
1.76 \\
0.47\end{array}$ \\
\hline VI & $\begin{array}{l}16 \mathrm{a} \\
17 \mathrm{~b} \\
18 \mathrm{c}\end{array}$ & $\begin{array}{l}0.890 \\
0.458 \\
0.187\end{array}$ & $\begin{array}{l}0.298 \\
1.953 \\
0.841\end{array}$ & $\begin{array}{l}0.728 \\
2.623 \\
1.165\end{array}$ & $\begin{array}{l}0.430 \\
0.670 \\
0.324\end{array}$ & $\begin{array}{l}1.87 \\
2.08 \\
0.88\end{array}$ & $\begin{array}{l}3.39 \\
3.16 \\
1.01\end{array}$ \\
\hline VII & $\begin{array}{l}19 \mathrm{a} \\
20 \mathrm{~b} \\
21 \mathrm{c}\end{array}$ & $\begin{array}{l}0.780 \\
0.567 \\
0.320\end{array}$ & $\begin{array}{l}0.991 \\
0.870 \\
0.365\end{array}$ & $\begin{array}{l}1.489 \\
1.224 \\
0.424\end{array}$ & $\begin{array}{l}0.498 \\
0.354 \\
0.059\end{array}$ & $\begin{array}{l}4.37 \\
2.26 \\
1.72\end{array}$ & $\begin{array}{l}7.66 \\
2.63 \\
1.94\end{array}$ \\
\hline VIII & $\begin{array}{l}22 \mathrm{a} \\
23 \mathrm{~b} \\
24 \mathrm{c}\end{array}$ & $\begin{array}{l}0.987 \\
0.564 \\
0.234\end{array}$ & $\begin{array}{l}0.361 \\
0.643 \\
0.291\end{array}$ & $\begin{array}{l}1.343 \\
1.399 \\
0.511\end{array}$ & $\begin{array}{l}0.982 \\
0.756 \\
0.220\end{array}$ & $\begin{array}{l}5.98 \\
1.22 \\
0.28\end{array}$ & $\begin{array}{l}8.65 \\
1.38 \\
0.32\end{array}$ \\
\hline IX & $\begin{array}{l}25 \mathrm{a} \\
26 \mathrm{~b} \\
27 \mathrm{c}\end{array}$ & $\begin{array}{l}0.567 \\
0.432 \\
0.229\end{array}$ & $\begin{array}{l}1.954 \\
0.879 \\
0.194\end{array}$ & $\begin{array}{l}2.386 \\
1.859 \\
0.425\end{array}$ & $\begin{array}{l}0.432 \\
0.980 \\
0.231\end{array}$ & $\begin{array}{l}4.70 \\
0.87 \\
1.14\end{array}$ & $\begin{array}{l}6.97 \\
1.47 \\
1.75\end{array}$ \\
\hline $\mathrm{X}$ & $\begin{array}{l}28 \mathrm{a} \\
29 \mathrm{~b} \\
30 \mathrm{c}\end{array}$ & $\begin{array}{l}0.402 \\
0.382 \\
0.287\end{array}$ & $\begin{array}{l}0.980 \\
0.328 \\
0.211\end{array}$ & $\begin{array}{l}1.745 \\
0.868 \\
0.234\end{array}$ & $\begin{array}{l}0.765 \\
0.540 \\
0.023\end{array}$ & $\begin{array}{l}0.72 \\
8.11 \\
2.56\end{array}$ & $\begin{array}{r}0.86 \\
10.96 \\
3.23\end{array}$ \\
\hline XI & $\begin{array}{l}31 \mathrm{a} \\
32 \mathrm{~b} \\
33 \mathrm{c}\end{array}$ & $\begin{array}{l}0.320 \\
0.469 \\
0.108\end{array}$ & $\begin{array}{l}0.529 \\
1.532 \\
0.851\end{array}$ & $\begin{array}{l}0.876 \\
2.512 \\
1.064\end{array}$ & $\begin{array}{l}0.347 \\
0.980 \\
0.213\end{array}$ & $\begin{array}{c}1.87 \\
12.3 \\
1.60\end{array}$ & $\begin{array}{l}2.40 \\
1.73 \\
1.08\end{array}$ \\
\hline XII & $\begin{array}{l}34 \mathrm{a} \\
35 \mathrm{~b} \\
36 \mathrm{c}\end{array}$ & $\begin{array}{l}0.980 \\
0.765 \\
0.309\end{array}$ & $\begin{array}{l}0.527 \\
0.265 \\
0.289\end{array}$ & $\begin{array}{l}1.096 \\
0.776 \\
0.609\end{array}$ & $\begin{array}{l}0.569 \\
0.511 \\
0.320\end{array}$ & $\begin{array}{l}9.45 \\
4.64 \\
2.89\end{array}$ & $\begin{array}{r}13.03 \\
7.08 \\
1.06\end{array}$ \\
\hline XIII & $\begin{array}{l}37 \mathrm{a} \\
38 \mathrm{~b} \\
39 \mathrm{c}\end{array}$ & $\begin{array}{l}0.387 \\
0.452 \\
0.320\end{array}$ & $\begin{array}{l}1.067 \\
0.829 \\
0.358\end{array}$ & $\begin{array}{l}1.806 \\
1.296 \\
0.708\end{array}$ & $\begin{array}{l}0.739 \\
0.467 \\
0.350\end{array}$ & $\begin{array}{r}19.96 \\
5.80 \\
3.98\end{array}$ & $\begin{array}{r}15.43 \\
0.94 \\
0.64\end{array}$ \\
\hline XIV & $\begin{array}{l}40 \mathrm{a} \\
41 \mathrm{~b} \\
42 \mathrm{c}\end{array}$ & $\begin{array}{l}0.321 \\
0.301 \\
0.254\end{array}$ & $\begin{array}{l}0.110 \\
0.321 \\
0.834\end{array}$ & $\begin{array}{l}0.950 \\
1.218 \\
0.849\end{array}$ & $\begin{array}{l}0.840 \\
0.897 \\
0.015\end{array}$ & $\begin{array}{r}11.89 \\
6.98 \\
2.06\end{array}$ & $\begin{array}{l}9.70 \\
1.25 \\
1.85\end{array}$ \\
\hline $\mathrm{XV}$ & $\begin{array}{l}43 \mathrm{a} \\
44 \mathrm{~b} \\
45 \mathrm{c}\end{array}$ & $\begin{array}{l}0.654 \\
0.432 \\
0.322\end{array}$ & $\begin{array}{l}0.231 \\
0.358 \\
0.389\end{array}$ & $\begin{array}{l}0.670 \\
0.738 \\
0.518\end{array}$ & $\begin{array}{l}0.439 \\
0.380 \\
0.129\end{array}$ & $\begin{array}{r}13.76 \\
4.87 \\
1.03\end{array}$ & $\begin{array}{l}7.27 \\
1.56 \\
0.23\end{array}$ \\
\hline XVI & $\begin{array}{l}47 \mathrm{a} \\
48 \mathrm{~b} \\
49 \mathrm{c}\end{array}$ & $\begin{array}{l}0.531 \\
0.632 \\
0.313\end{array}$ & $\begin{array}{l}0.320 \\
0.540 \\
0.371\end{array}$ & $\begin{array}{l}0.790 \\
0.851 \\
0.477\end{array}$ & $\begin{array}{l}0.470 \\
0.311 \\
0.106\end{array}$ & $\begin{array}{r}9.85 \\
12.54 \\
2.76\end{array}$ & $\begin{array}{l}3.20 \\
2.93 \\
0.97\end{array}$ \\
\hline XVII & $\begin{array}{l}50 \mathrm{a} \\
51 \mathrm{~b} \\
52 \mathrm{c}\end{array}$ & $\begin{array}{l}0.431 \\
0.219 \\
0.201\end{array}$ & $\begin{array}{l}0.298 \\
1.012 \\
0.876\end{array}$ & $\begin{array}{l}1.118 \\
1.332 \\
1.263\end{array}$ & $\begin{array}{l}0.820 \\
0.320 \\
0.387\end{array}$ & $\begin{array}{r}16.33 \\
7.83 \\
3.63\end{array}$ & $\begin{array}{l}6.39 \\
2.52 \\
1.64\end{array}$ \\
\hline XVIII & $\begin{array}{l}53 \mathrm{a} \\
54 \mathrm{~b} \\
55 \mathrm{c}\end{array}$ & $\begin{array}{l}0.438 \\
0.576 \\
0.309\end{array}$ & $\begin{array}{l}0.678 \\
0.459 \\
0.769\end{array}$ & $\begin{array}{l}1.135 \\
1.133 \\
1.002\end{array}$ & $\begin{array}{l}0.457 \\
0.674 \\
0.233\end{array}$ & $\begin{array}{l}7.93 \\
3.86 \\
2.64\end{array}$ & $\begin{array}{l}8.47 \\
1.68 \\
0.25\end{array}$ \\
\hline
\end{tabular}


Table 2. Continued

\begin{tabular}{|c|c|c|c|c|c|c|c|}
\hline Patient no. & Tissue sample & $\mathrm{CP}(\mathrm{Col})$ & $\mathrm{ICP}^{37}$ & $\mathrm{ICP}^{80}$ & $\Delta \mathrm{ICP}$ & $\mathrm{CP}(\mathrm{F})$ & $\mathrm{pCP}(\mathrm{F})$ \\
\hline XIX & $\begin{array}{l}56 \mathrm{a} \\
57 \mathrm{~b} \\
58 \mathrm{c}\end{array}$ & $\begin{array}{l}0.719 \\
0.812 \\
0.087\end{array}$ & $\begin{array}{l}0.348 \\
0.278 \\
0.304\end{array}$ & $\begin{array}{l}0.695 \\
0.563 \\
0.407\end{array}$ & $\begin{array}{l}0.347 \\
0.285 \\
0.103\end{array}$ & $\begin{array}{r}12.75 \\
5.79 \\
4.98\end{array}$ & $\begin{array}{l}7.11 \\
1.67 \\
2.20\end{array}$ \\
\hline XX & $\begin{array}{l}59 a \\
60 \mathrm{~b} \\
61 \mathrm{c}\end{array}$ & $\begin{array}{l}0.689 \\
0.430 \\
0.301\end{array}$ & $\begin{array}{l}1.967 \\
0.876 \\
0.996\end{array}$ & $\begin{array}{l}2.610 \\
1.399 \\
1.253\end{array}$ & $\begin{array}{l}0.643 \\
0.523 \\
0.257\end{array}$ & $\begin{array}{l}7.33 \\
4.76 \\
2.32\end{array}$ & $\begin{array}{r}3.66 \\
12.87 \\
0.88\end{array}$ \\
\hline XXI & $\begin{array}{l}62 \mathrm{a} \\
63 \mathrm{~b} \\
64 \mathrm{c}\end{array}$ & $\begin{array}{l}0.597 \\
0.601 \\
0.321\end{array}$ & $\begin{array}{l}0.560 \\
0.398 \\
0.329\end{array}$ & $\begin{array}{l}1.492 \\
0.756 \\
0.562\end{array}$ & $\begin{array}{l}0.932 \\
0.358 \\
0.233\end{array}$ & $\begin{array}{l}5.87 \\
3.97 \\
0.96\end{array}$ & $\begin{array}{l}2.75 \\
7.66 \\
1.02\end{array}$ \\
\hline XXII & $\begin{array}{l}65 \mathrm{a} \\
66 \mathrm{~b} \\
67 \mathrm{c}\end{array}$ & $\begin{array}{l}0.760 \\
0.450 \\
0.296\end{array}$ & $\begin{array}{l}1.071 \\
0.894 \\
0.692\end{array}$ & $\begin{array}{l}1.653 \\
1.731 \\
0.923\end{array}$ & $\begin{array}{l}0.582 \\
0.837 \\
0.231\end{array}$ & $\begin{array}{r}16.76 \\
3.76 \\
9.63\end{array}$ & $\begin{array}{r}5.74 \\
11.23 \\
3.53\end{array}$ \\
\hline XXIII & $\begin{array}{l}68 \mathrm{a} \\
69 \mathrm{~b} \\
70 \mathrm{c}\end{array}$ & $\begin{array}{l}0.583 \\
0.210 \\
0.071\end{array}$ & $\begin{array}{l}0.651 \\
1.076 \\
0.873\end{array}$ & $\begin{array}{l}1.218 \\
1.861 \\
0.971\end{array}$ & $\begin{array}{l}0.567 \\
0.785 \\
0.098\end{array}$ & $\begin{array}{r}12.31 \\
19.51 \\
1.98\end{array}$ & $\begin{array}{l}6.87 \\
7.54 \\
4.74\end{array}$ \\
\hline XXIV & $\begin{array}{l}71 \mathrm{a} \\
72 \mathrm{~b} \\
73 \mathrm{c}\end{array}$ & $\begin{array}{l}0.349 \\
0.356 \\
0.207\end{array}$ & $\begin{array}{l}0.349 \\
0.480 \\
0.312\end{array}$ & $\begin{array}{l}0.881 \\
1.391 \\
0.631\end{array}$ & $\begin{array}{l}0.532 \\
0.911 \\
0.319\end{array}$ & $\begin{array}{l}4.75 \\
3.99 \\
0.74\end{array}$ & $\begin{array}{l}6.33 \\
5.35 \\
2.87\end{array}$ \\
\hline XXV & $\begin{array}{l}74 \mathrm{a} \\
75 \mathrm{~b} \\
76 \mathrm{c}\end{array}$ & $\begin{array}{l}0.861 \\
0.398 \\
0.200\end{array}$ & $\begin{array}{l}1.073 \\
0.753 \\
0.590\end{array}$ & $\begin{array}{l}1.349 \\
1.589 \\
0.693\end{array}$ & $\begin{array}{l}0.276 \\
0.836 \\
0.103\end{array}$ & $\begin{array}{r}13.22 \\
7.92 \\
2.43\end{array}$ & $\begin{array}{l}2.98 \\
3.59 \\
2.84\end{array}$ \\
\hline XXVI & $\begin{array}{l}76 \mathrm{a} \\
77 \mathrm{~b} \\
78 \mathrm{c}\end{array}$ & $\begin{array}{l}0.471 \\
0.318 \\
0.197\end{array}$ & $\begin{array}{l}0.378 \\
0.210 \\
0.386\end{array}$ & $\begin{array}{l}0.908 \\
0.972 \\
0.707\end{array}$ & $\begin{array}{l}0.530 \\
0.762 \\
0.321\end{array}$ & $\begin{array}{l}8.82 \\
7.43 \\
6.86\end{array}$ & $\begin{array}{l}3.75 \\
5.87 \\
3.85\end{array}$ \\
\hline XXVII & $\begin{array}{l}79 \mathrm{a} \\
80 \mathrm{~b} \\
81 \mathrm{c}\end{array}$ & $\begin{array}{l}0.542 \\
0.498 \\
0.295\end{array}$ & $\begin{array}{l}0.476 \\
0.531 \\
0.379\end{array}$ & $\begin{array}{l}1.147 \\
0.878 \\
0.598\end{array}$ & $\begin{array}{l}0.671 \\
0.347 \\
0.219\end{array}$ & $\begin{array}{r}13.25 \\
11.37 \\
1.24\end{array}$ & $\begin{array}{l}3.22 \\
4.96 \\
1.07\end{array}$ \\
\hline XXVIII & $\begin{array}{l}76 \mathrm{a} \\
77 \mathrm{~b} \\
78 \mathrm{c}\end{array}$ & $\begin{array}{l}0.431 \\
0.397 \\
0.254\end{array}$ & $\begin{array}{l}0.396 \\
0.365 \\
0.254\end{array}$ & $\begin{array}{l}0.915 \\
0.862 \\
0.600\end{array}$ & $\begin{array}{l}0.519 \\
0.497 \\
0.346\end{array}$ & $\begin{array}{r}13.25 \\
11.37 \\
6.21\end{array}$ & $\begin{array}{l}3.01 \\
6.16 \\
0.22\end{array}$ \\
\hline XXIX & $\begin{array}{l}79 \mathrm{a} \\
80 \mathrm{~b} \\
81 \mathrm{c}\end{array}$ & $\begin{array}{l}0.349 \\
0.307 \\
0.287\end{array}$ & $\begin{array}{l}1.042 \\
0.983 \\
1.034\end{array}$ & $\begin{array}{l}1.481 \\
1.268 \\
1.160\end{array}$ & $\begin{array}{l}0.439 \\
0.285 \\
0.126\end{array}$ & $\begin{array}{r}15.98 \\
4.85 \\
2.09\end{array}$ & $\begin{array}{l}8.52 \\
6.74 \\
0.62\end{array}$ \\
\hline
\end{tabular}

(a) center of tumor, (b) zone of invasion, and (c) healthy tissue

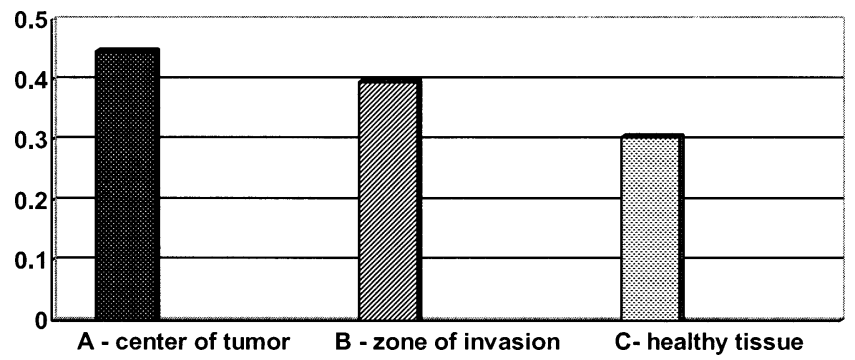

Fig. 1. Mean activities of cysteine peptidases (CP) in three different types of tissue samples in gastric cancer, as determined by colorimetry $(\mathrm{Col})$

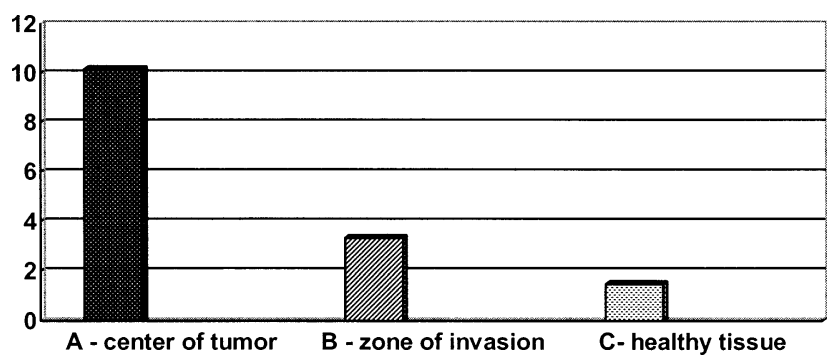

Fig. 2. Mean activities of cysteine peptidases (CP) in three different types of tissue samples in gastric cancer, as determined by spectrofluorometry $(\mathrm{F})$ 


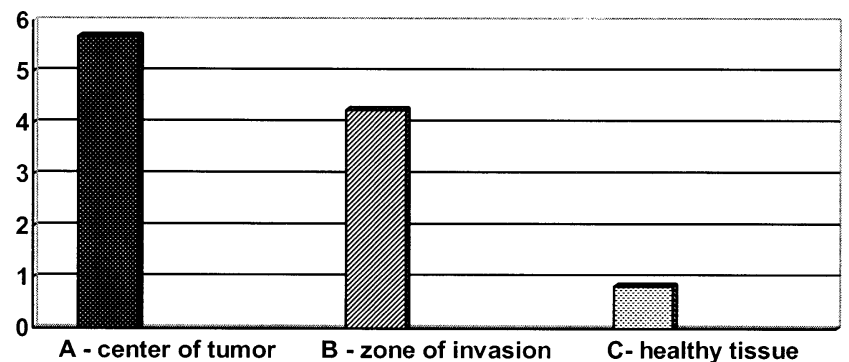

Fig. 3. Mean activities of precursors of cysteine peptidases (pCP) in three different types of tissue samples in gastric cancer

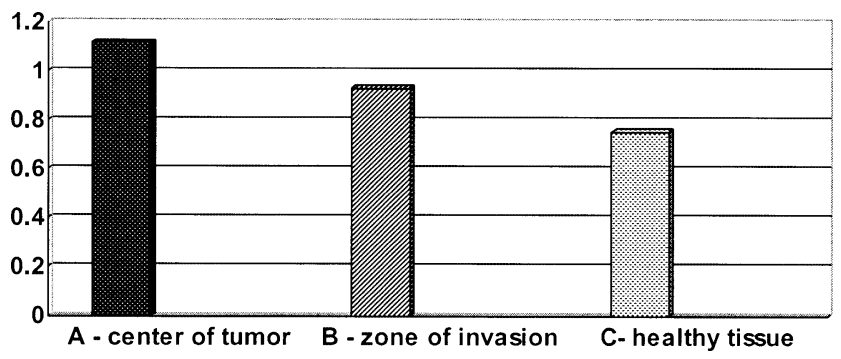

Fig. 4. Mean activities of active inhibitors of cysteine peptidases $\left(\mathrm{ICP}^{37}\right)$ in three different types of tissue samples in gastric cancer

\section{Discussion}

The reported relationships between selected proteolytic enzymes and the aggressiveness of malignant tumors indicated possible changes in the activities of cysteine peptidases and their autogenous inhibitors in the homogenates of tissues obtained from patients operated on for gastric cancer. To date, cathepsins $\mathrm{B}$ and $\mathrm{L}$ have usually been measured by enzyme-linked immunosorbent assay (ELISA) in the tissues and serum of patients with gastrointestinal cancers [16]. Such assessments of cathepsins B and $\mathrm{L}$ in tissues resected during operations for gastric cancer had shown maximum concentration in the tumor mass, while in the surrounding healthy tissue these enzymes were less abundant. This confirmed the previous assumptions that cysteine peptidases may play a key role in the processes accompanying malignant growth. Our study was intended to provide supplementary information on changes in enzymatic activity in tumor growth. The measurements of precursors of cathepsins $\mathrm{B}$ and $\mathrm{L}$ provided information about the potential of malignant tumor expansion. Activation of these precursors is an indicator of enhanced potential of tumor growth. On the other hand, the activity of inhibitors of these enzymes indicates the organism's defense ability against cancer. In the samples analyzed in this study, we measured three

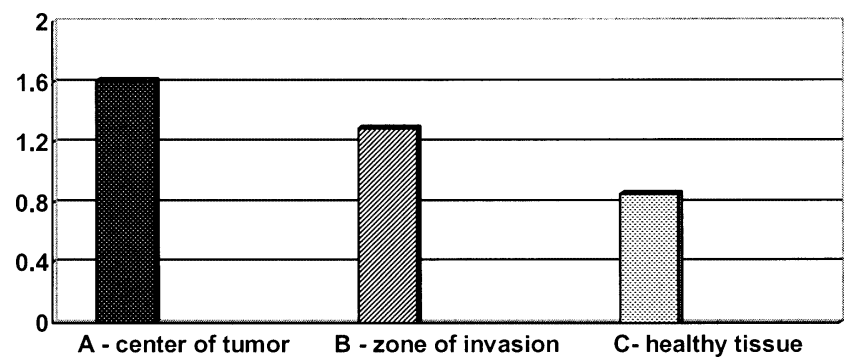

Fig. 5. Mean activities of total pool of cysteine peptidase inhibitors $\left(\mathrm{ICP}^{80}\right)$ in three different types of tissue samples in gastric cancer

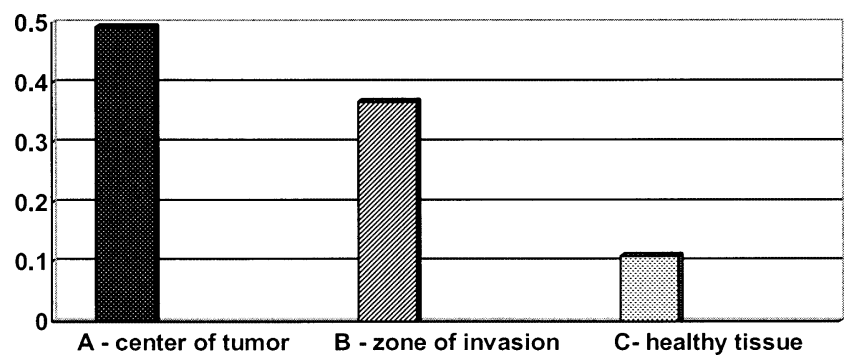

Fig. 6. Mean activities of cysteine peptidase inhibitors bound in enzyme-inhibitor complexes $(\triangle \mathrm{ICP})$ in three different types of tissue samples in gastric cancer

forms of specific inhibitors of cysteine peptidases: active inhibitors, the total pool of inhibitors produced, and the inhibitors bound in enzyme-inhibitor complexes. The active inhibitors were characterized by the ability to inhibit papain, which is a cysteine peptidase.

The inhibitors complexed with cysteine peptidases, which are secreted by tumor cells, we considered one of the markers of malignancy, and we referred to them as $\triangle \mathrm{ICP}[9]$. Gastric cancer was intentionally selected as the subject of our study, considering the acidic $\mathrm{pH}$ and the presence of pepsin in the stomach, which can activate the precursors of cysteine peptidases to the active form [4]. We surmise that the observed differences between the tissues sampled from the tumor mass, the zone of invasion, and the normal tissue within the same patient with gastric cancer result from the action of pepsin at low $\mathrm{pH}$, which directly affects the tissues in which the malignant tumor is growing, thus catalyzing the transformation of cathepsin $\mathrm{B}$ and $\mathrm{L}$ precursors to the active enzymes, which in turn, enhances the tumor's invasiveness. Our results for the measured activities of $\mathrm{CP}, \mathrm{pCP}$, and $\triangle \mathrm{ICP}$ suggest that these parameters may prove to be a useful diagnostic tool for evaluating the dynamics of malignant growth within the tissues of gastric cancer. In the future, these parameters may play some role in the designing of the extent of the operation. To elucidate this we are planning a similar study 
with respect to bioptates taken endoscopically before the operation. Other important information implied by previous studies is that there may be some possibility of treating gastric cancer with specific inhibitors of cysteine peptidases, isolated from egg white. So far we have been able to demonstrate the inhibition of cysteine peptidases which initiate the growth of gastric cancer only in vitro, in homogenates of tissues sampled from resected material after operation [17]. The information that cysteine peptidase inhibitors isolated from egg whites are able to inhibit the elevated activity of cysteine endopeptidases in gastric cancer cells suggests that these inhibitors might be used as a component of newgeneration drugs in so-called "inhibitor therapy" [18]. These inhibitors have been proved nontoxic and may be administered orally. In addition, there is a possibility of labelling them with fluorescent agents approved for medical use, which might be helpful in UV evaluation of the extent of malignant infiltration [19]. There are also prospects for introducing a new method of therapy that would consist of cysteine proteinase inhibitor therapy combined with photodynamic therapy [20].

Moreover, antibodies against these inhibitors may be used for detecting the activity of cysteine peptidases in preoperative endoscopic bioptates, which could be helpful in planning the extent of the operation [21]. In 2002 we found that, in tissue cultures of malignant tumors, cysteine peptidase inhibitors labelled with radioactive iodine reacted selectively, only with cancer cells [22]. So far these investigations have been limited to in vitro experiments, but at the moment we are preparing a series of experiments which might allow further studies in vivo, at first on animals; we hope that in the future, such studies could be carried out in gastric cancer patients.

\section{Conclusions}

1. Local activities of cysteine peptidases and their inhibitors reflect the topographical differences between the center of the tumor, the zone of invasion, and healthy tissues in gastric cancer patients.

2. The findings of the pattern of changes in the activity of cysteine peptidases according to the degree of tissue infiltration are not dependent on the method of measurement (colorimetry vs spectrofluorometry).

\section{References}

1. Plebani M, Herszenyi L, Cardin R, Roveroni G, Carraro P, Paoli $\mathrm{MD}$, et al. Cysteine and serine proteases in gastric cancer. Cancer 1995;76:367-75.
2. Herszényi L, Farinati F, Plebani M, Carraro P, Roveroni G, De Paoli M, et al. Prognostic role of cysteine and serine proteases in gastric cancer. Orv Hetil 1996;137:1637-41.

3. Schmitt M, Janicke F, Graeff H. Tumor-associated proteases. Fibrynolysis 1992;6:3-26.

4. Pagano M, Capony F, Rochefort H. Pro-cathepsin D can activate in vitro procathepsin B secreted by ovarian cancers. C R Acad Sci III 1989;309:7-12.

5. Maciewicz RA, Wardale RJ, Wotton SF, Duance VC, Etherington DJ. Mode of activation of the precursor to cathepsin L: implication for matrix degradation in arthritis. Biol Chem Hoppe Seyler 1990;371:223-30.

6. Mion K, Cao L, Day NA, Koblinski JE, Sloane BF. Tumor cell membrane cathepsin B. Biol Chem 1998;379:1093-9.

7. Laszlo A, Sohar I, Karacsonyi-S, Petri A, Trojan I. Activities of serum cathepsin (B, H and L) and metalloproteinase (MMP7-ase) in patients with gastrointestinal and bronchial malignant tumours. Acta Med Hung 1990;47:107-9.

8. Sheahan K, Shuja S, Murnane MJ. Cysteine protease activities and tumor development in human colorectal carcinoma. Cancer Res 1989;49:3809-14.

9. Siewinski M, Gutowicz J, Mikulewicz W, Zarzycki A. Participation of cysteine endopeptidases in the invasion and metastasis of tumor and in neoplastic transformation. Cancer Biotherapy Pharmaceuticals 1996;11:321-31.

10. Dohchin A, Suzuki J, Seki H, Masutani M, Shiroto H, Kawakami Y. Immunostained cathepsins B and L correlate with depth of invasion and different metastatic pathways in early stage gastric carcinoma. Cancer 2000;89:482-7.

11. Barrett AJ. Fluorimetric assays for cathepsin B and cathepsin $\mathrm{H}$ with methylcoumarylamide substrates. Biochem J 1980;187:90912.

12. Krecicki T, Siewinski M. Serum cathepsin B-like activity as a potential marker of laryngeal carcinoma. Eur Arch Otorhinolaryngol 1992;249:293-5.

13. Minakata K, Asano M, Sato T, Harada N. Assay of a cysteine inhibitor in serum or plasma. Hoppe Seyler's Z Physiol Chem 1982;363:493-8.

14. Siewinski M, Krecicki T, Berdowska I, Jarmulowicz J. Cysteine proteinase inhibitors in serum of patients with head and neck tumors. Diagn Oncol 1993;2:323-6.

15. Bradford MM. A rapid and sensitive method for quantitation of microgram quantities of protein utilizing the principal of proteindye binding. Anal Biochem 1976;72:248-54.

16. Farinati F, Herszényi L, Plebani M, Carraro P, De Paoli M, Cardin R, et al. Increased levels of cathepsin B and L, urokinasetype plasminogen activator and its inhibitor type- 1 as an early event in gastric carcinogenesis. Carcinogenesis 1996;17:2581-7.

17. Saleh Y, Siewinski M, Kielan W. Inhibition in vitro of cysteine endopeptidases-like activity in stomach carcinoma tissues. Cancer Detect Prev 2002;26:1291.

18. Lah TT, Kos J. Cysteine proteinases in cancer progression and their clinical relevance for prognosis. Biol Chem 1998;379:12530 .

19. Gutowicz J, Michalak K, Pola A, Berdowska I, Siewinski M. Fluorescence labelling of thiol proteinase inhibitors excreted from urine of colorectal cancer patients. Current Topics in Biophysics 1996;21:238-45.

20. Saleh Y, Ziólkowski P, Siewinski M, Milach J, Marszalik P, Rybka J. The combined therapy of transplantable solid mammary carcinoma in Wistar rats by use of photodynamic therapy and cysteine proteinase inhibitors. In Vivo 2001;15:351-8.

21. Siewinski M, Saleh Y, Popiela A, Ziólkowski P, Jelen M, Grybos M. Expression of high molecular weight cysteine proteinase inhibitor in ovarian cancer tissues: regulation of cathepsin B expression by placental CPL. Biol Chem 2003;384:1103-7.

22. Czecior E, Szymaniec S, Siewinski M, Fortuna W, Miedzybrodzki W. A potential use of cysteine endopeptidases inhibitors of upper respiratory tract tumors. Otolaryngol Pol 2002;56:573-6. 